\title{
Automatic Inspection System for Electric Motors with Integrated Bus Interface
}

\author{
András Lelkes \\ GEFEG-NECKAR Antriebssysteme GmbH, Gosheim, D-78559, Germany
}

\begin{abstract}
This paper describes an automatic test system for small power electric motors with integrated intelligent control unit. The testing concept utilizes the sensors integrated in the motor and the communication capabilities of the drive. Therefore, the motors can be tested quickly, yet thoroughly during manufacturing without the need for cost intensive test stands.
\end{abstract}

Keywords Electric Machines, Variable Speed Drives, Field Bus, Automatic Test Equipment, Quality Management

\section{Introduction}

The company GEFEG-NECKAR Antriebssysteme is successor of the motor manufacturers Gefeg, founded in 1948, and Neckar Kleinstmotoren, founded in 1967. The latter produced compact brushless dc motors with integrated electronic motor control since 1995.

In 2005, the merged company started the development of a new electronic platform with the capability of bus communication. Feedback from customers showed that CANopen ${ }^{\circledR}$ was a good choice for a high-performance but cost-effective bus solutions.

The integration of motor and control unit makes elegant and compact drive solutions for numerous industrial applications possible. The drives decrease wiring effort in the system and space requirement in the electronics cabinet. Additionally, the integrated bus interface of these motors enables an efficient automatic final test in the motor production line without the need for cost intensive test stands.

\section{Compact Drives with Integrated CAN Interface}

The first application of the new, bus capable electronic platform has been a compact BLDC drive. These motors have long service life as a result of the brushless technique, high efficiency by high-quality rare earth magnets and high environmental protecting class by compact, closed construction (Figure 1). These motors can be combined, similar to all motors of this manufacturer, with worm, spur and planetary gears, with brakes and with shaft encoders.

* Corresponding author:

a.lelkes@gefeg-neckar.de (Dr. András Lelkes)

Published online at http://journal.sapub.org/eee

Copyright (C) 2012 Scientific \& Academic Publishing. All Rights Reserved
The control algorithm is implemented in a low-cost DSP controller (dsPIC33F). The rotor position is detected by three Hall sensors. This position information serves for proper electronic commutation of the brushless motor. Additionally, the Hall sensors are used for speed measurement and for closed-loop speed control. The $24 \mathrm{~V}$ power module is based on SMD-MOSFET transistors. They are soldered on a PCB from aluminum enabling proper cooling.

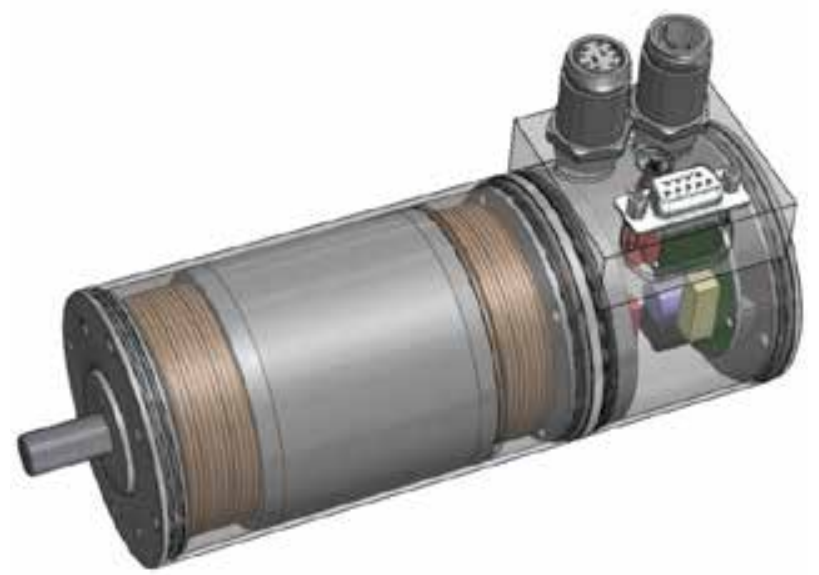

Figure 1. Brushless motor with integrated CANopen ${ }^{\circledR}$ interface (MC 663)

The CAN interface provides for bus communication using the CANopen ${ }^{\circledR}$ protocol (Drive Profile DSP-402). Customers not operating a bus system may still control the motor via analog or digital set point signals. The integrated bus communication provides certain benefits here, too, since it makes easy testing and parameter setting of the drives possible. The firmware also includes a boot loader. Therefore, firmware update can be easily done trough the CANopen ${ }^{\circledR}$ interface.

\subsection{Development of a New BLDC Motor Family}

By optimizing the magnet circuit and by implementation of a new winding technology, a new high-performance 
high-efficiency BLDC motor family was developed. The first member of this family is a compact brushless DC motor with a diameter of $68 \mathrm{~mm}$ (MQ 667, Figure 2).

In comparison to its ancestor with the same diameter ( $\mathrm{M}$ $663)$, axial length could be decreased by $15 \%$, torque increased by $60 \%$ and cogging torque reduced considerably.

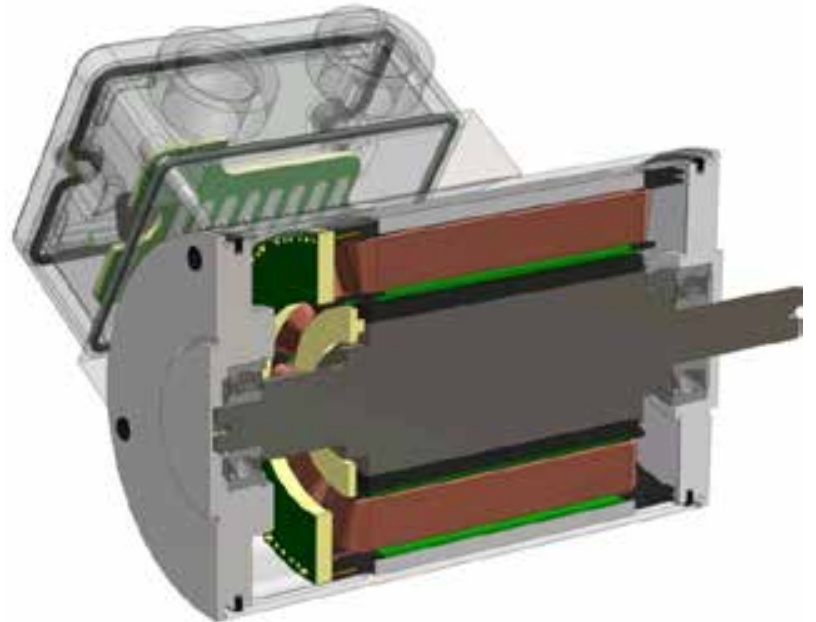

Figure 2. Newly developed high-performance BLDC motor (MQ 667)

Motors of the new family will be available with integrated CAN interface, too.

\subsection{PMDC motors with integrated CAN interface}

The electronic platform with CAN interface can be used to control permanent magnet DC motors, too. In spite of mechanical commutation, these drives also contain Hall sensors for the position detection. These sensors deliver speed information for the closed-loop speed control.

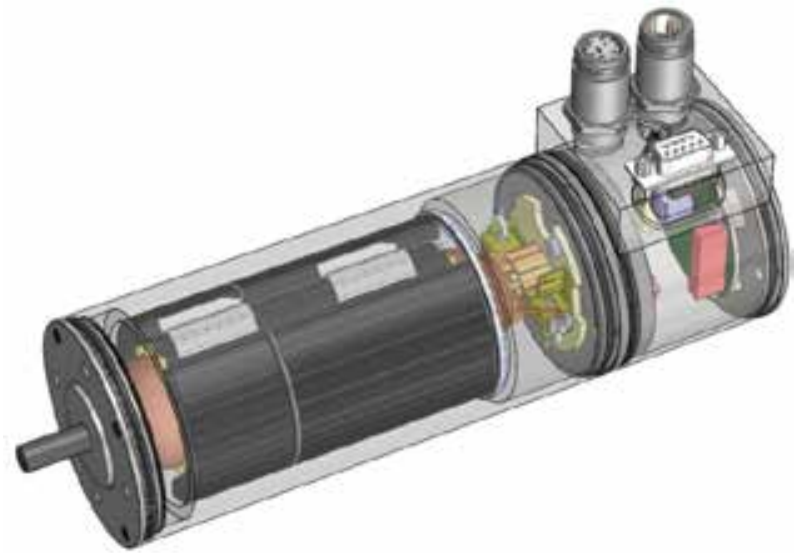

Figure 3. Permanent magnet DC motor with integrated CAN interface (PC 6355)

Due to inexpensive magnet systems (sintered ferrite segments) and automatic production lines, DC motors are very cost effective. On the other side, the life expectancy of these motors is limited by the commutation system consisting of collector and graphite brushes. However, there are numerous applications where the necessary active service hours do not exceed the maximum limit, in the majority of cases 3,000 hours. In such applications, a conventional DC motor with integrated speed control and bus interface might prove to be an interesting and cost effective alternative (Figure 3).

\subsection{External Motor Control Unit with Integrated CAN Interface}

By integrating all components of the system such as power unit, motor control unit, measurement system and bus interface, wiring and planning effort is reduced to a minimum. Of the elaborate wiring system for the individual components, only the supply cable and the field bus cable remain. Therefore, the availability of the system increases. Furthermore, the space required in the electronics cabinet is reduced. Nevertheless, there are applications where the integrated solution is not preferable. The reason can be that environmental temperature is too high or that installation space is strongly limited.

To cover such applications, an external control unit with CAN interface was developed (Figure 4). This control unit is based virtually on the same hardware and firmware as used in the integrated solutions. In doing so, the platform allows the users to control PMDC and BLDC motors in the same way - no matter whether the control unit is located in a switch cabinet or integrated into the motor.

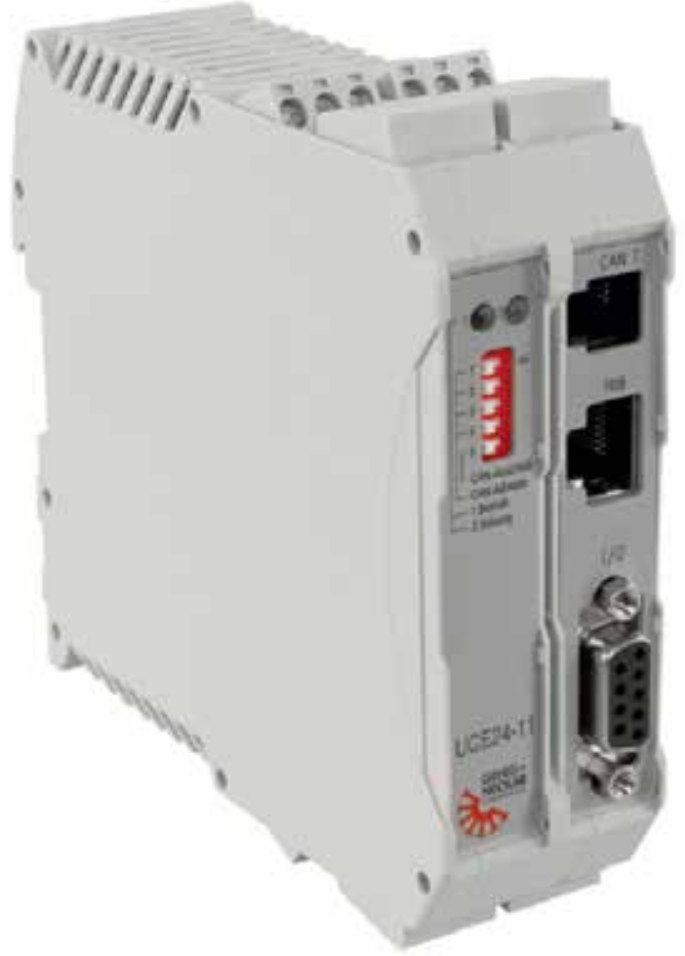

Figure 4. External unit with integrated CAN interface for controlling BLDC and PMDC motors (UCE24-11)

\subsection{Electronically Commutated Motors for $230 \mathrm{~V} 50 \mathrm{~Hz}$ Mains}

At safety low voltage drives, the AC mains voltage has to be converted in two steps. A switch-mode AC/DC converter generates a safety-low voltage (e.g. $24 \mathrm{~V}$ ) from the $230 \mathrm{~V}$ $50 \mathrm{~Hz}$ AC voltages. From this DC voltage, the commutation 
unit generates a three-phase AC voltage system with variable amplitude and frequency for the motor windings.

Using a commutation unit for direct mains operation, investment spending, system complexity and power dissipation can be reduced. For this reason, such commutation electronics were developed based on an intelligent power module (Figure 5).

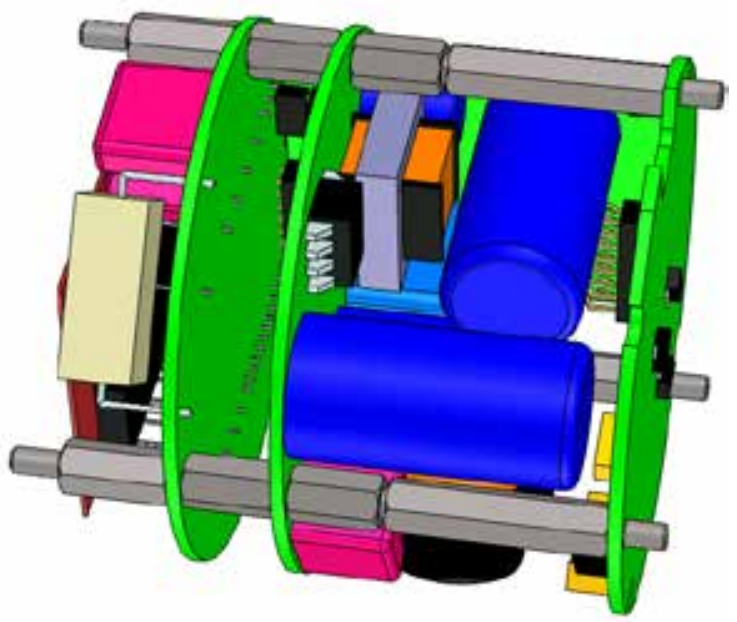

Figure 5. Commutation unit with CAN interface for direct $230 \mathrm{~V} / 50 \mathrm{~Hz}$ supply

In comparison to a safety low voltage DC unit, additional functional blocks have to be integrated:

- single phase bridge rectifier

- DC link capacitor

- initial current limiter

- EMC filter

- Switched mode DC/DC converter supplying the internal control electronics

- Safe potential separation between power circuits and interface signals

The difficulty of this task lay in designing a very compact unit while fulfilling the statutory low voltage directive and EMC regulations. An additional problem was how to resolve effective cooling to guarantee a reliable operation of the power and control electronics and to ensure a sufficient life span of the temperature-sensitive electrolytic dc link capacitors.

By integrating this electronic unit in a motor with permanent magnet rotor, an electronically commutated (EC) motor with integrated CAN interface for $230 \mathrm{~V} 50 \mathrm{~Hz}$ supply has been developed (Figure 6).

\subsection{Induction Motor with Integrated CAN Interface}

Induction motors have a lower nominal torque and a lower level of efficiency than electronically commutated motors do. However, induction motors do not need high prized rare earth magnets for their production. For speed variable drives, the induction motor can be supplied by a converter with variable frequency.

With a modified firmware, the commutation unit of the EC motor can act as a frequency converter for three-phase induc- tion motors. By integrating this unit into the motor housing, a very compact variable speed induction drive can be achieved (Figure 7).

Benefits of this drive are bus capability (CANopen ${ }^{\circledR}$ ) and closed-loop speed control, similar to those of all the other members of the large family of drives with the same electronic platform.
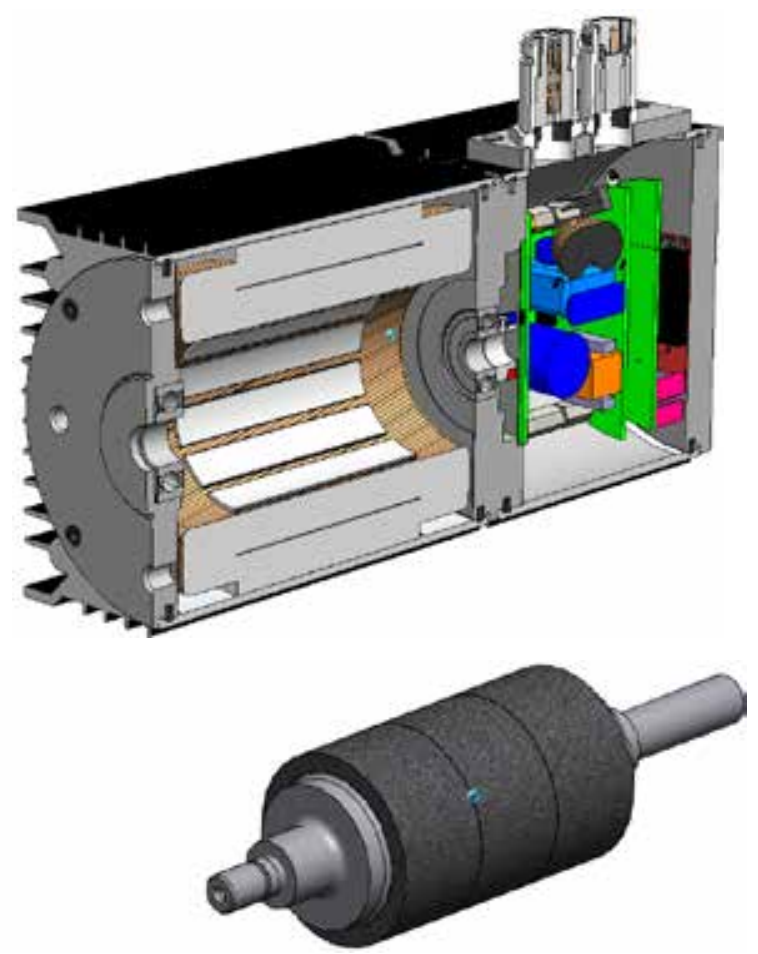

Figure 6. EC motor with integrated CAN interface for $230 \mathrm{~V} / 50 \mathrm{~Hz}$ supply (MCN 963)
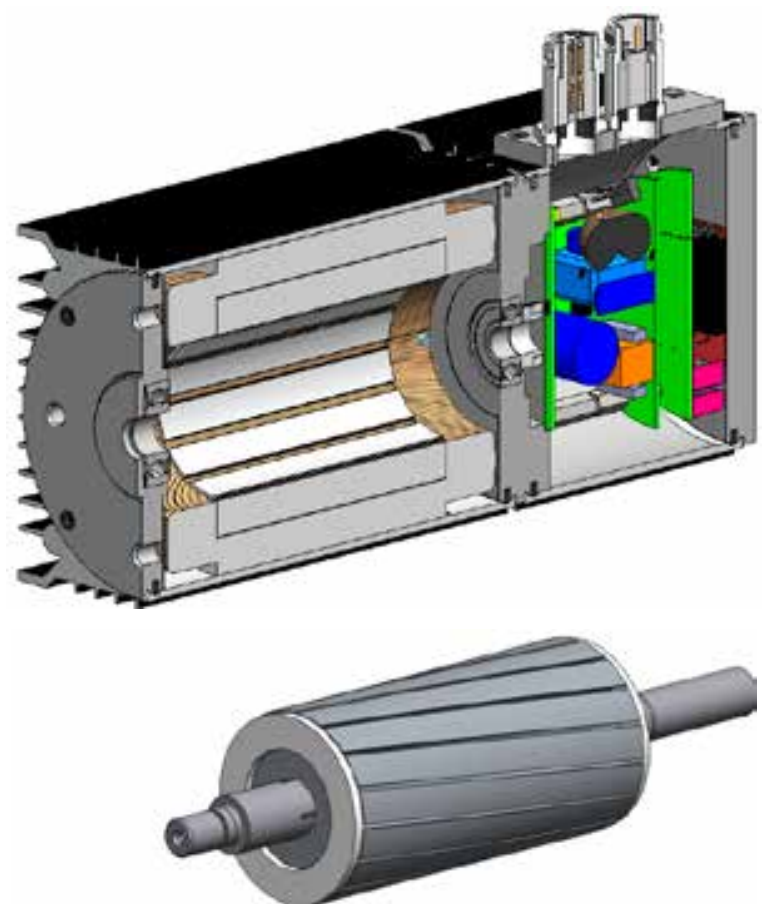

Figure 7. Variable speed induction drive with integrated CAN interface (ACN 983) 
All said drives offer a high degree of environmental protection. They can be combined with brakes and different gears. Furthermore, the unified electronic platform in these products allows new advantageous test procedures in the production and easy parameter settings by customers.

\section{Test System for Integrated Drives}

\subsection{Software for Manual Testing}

For manual testing of the motors with integrated control unit and bus interface, the PC software "MotorMonitor" for Windows ${ }^{\circledR}$ based computers has been developed. The software is written in the programming language $\mathrm{C \#}$. It needs only a CAN/USB adapter to communicate with the motor via CANopen ${ }^{\circledR}$ protocol (Drive Profile DSP-402).

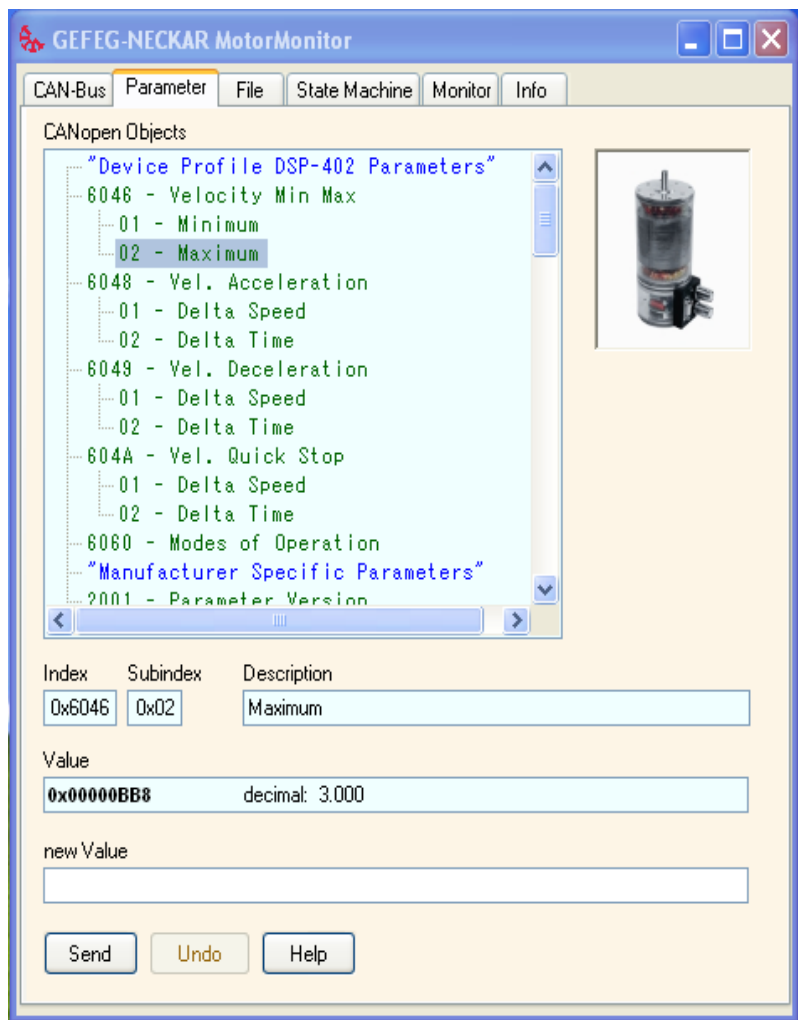

Figure 8. Graphical user interface of the commissioning software "MotorMonitor", tab card "Parameter"

At first, the computer program builds up the communication with the motor. After that, it requests the firmware version of the drive. The software contains a data base with all released firmware versions. Therefore, only the parameters implemented in the actual motor are displayed.

The software allows the user to change parameters (Figure 8), control the drive (Figure 9) or monitor important data such as rotor position, velocity, power consumption, and temperature.

For commissioning, parameter setting, manual testing and monitoring, the company grants rights of use of this software to its customers free of charge. However, customers cannot change certain safety-relevant parameters.

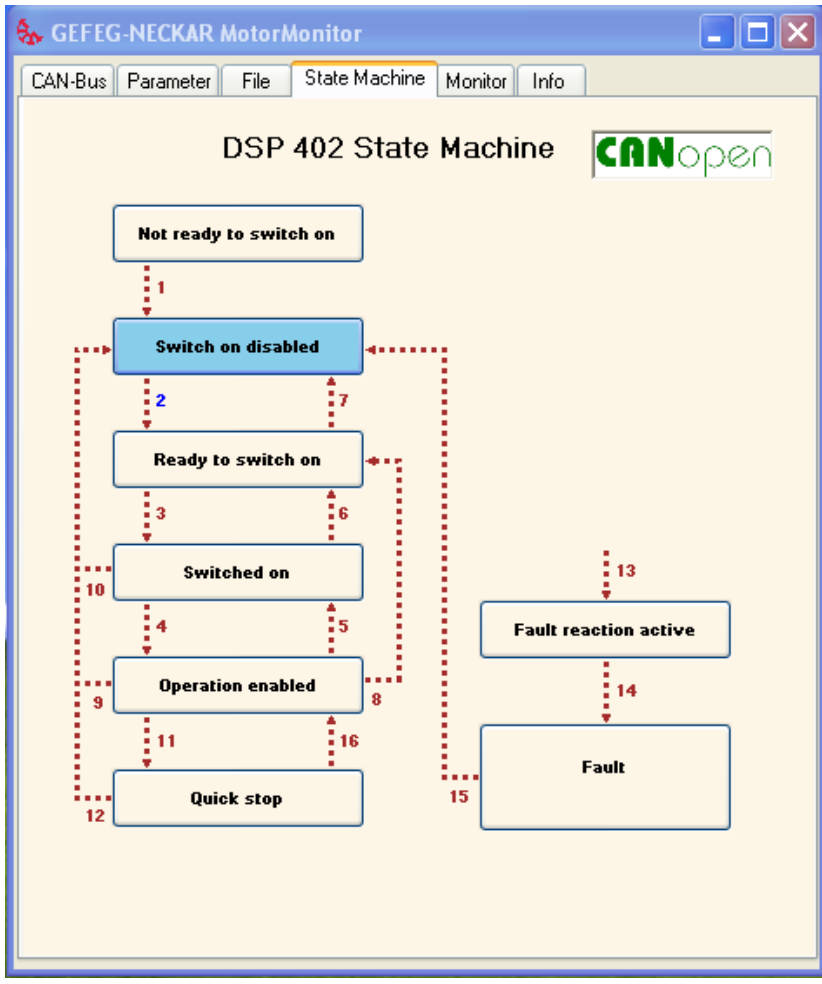

Figure 9. Tab card "State Machine" of the commissioning software

\subsection{Automatic inspection system integrated in the pro- duction process}

The integrated bus capable electronic control unit enables an easy, cost effective but detailed test of these motors in the production line. For the final test, a new inspection system has been developed. It utilizes a standardized protocol to communicate with the integrated electronic platform (CANopen ${ }^{\circledR}$ Drive Profile DSP 402). The test program is based on the routines of the commissioning software "MotorMonitor"

Without requiring any expensive test station, it is now possible to test the drives automatically. There is no need for any additional sensors for testing, because the integrated intelligent control unit contains all the sensors needed for an adequate test (Figure 10).

\begin{tabular}{|l|l|}
\hline \multicolumn{1}{|c|}{ Sensor } & \multicolumn{1}{c|}{ Purpose } \\
\hline Hall-sensors & $\begin{array}{l}\text { - Rotor position detection } \\
\text { - Electronic commutation } \\
\text { - Speed measurement }\end{array}$ \\
\hline Voltage sensor & $\begin{array}{l}\text { - Over voltage protection } \\
\text { - Under voltage protection } \\
\text { - Brake circuit control }\end{array}$ \\
\hline Current sensor & - Over current protection \\
\hline Temperature sensors & $\begin{array}{l}\text { - Over temperature protection } \\
\text { (motor / power electronics) }\end{array}$ \\
\hline
\end{tabular}

Figure 10. Integrated sensors of the control unit

The test computer communicates with the microcontroller in motor through the CAN interface and receives the measured values (supply voltage, motor current, rotor position, speed, and temperature).

The test computer itself is integrated into the computer 
system of the production facility. The production planning and control system (PPC) generates updated files containing the current released production orders (job account number, tracking number, internal part number, product name, and production volume).

The tester adds his or her name to the protocol and scans the bar code of the production document. After that, the test computer identifies the drive in the production order file. For every product, three specific ASCII files are prepared and stored in the test system:

- test procedure and expected test results

- rating plate data

- parameter data at delivery

For that reason, there is no need to type in any further information. The operator can select the test mode (test run or recorded product test with setting of parameters and printing of rating plate) and start the test (Figure 11).

In the first step of test (Figure 12), the CANopen ${ }^{\circledR}$ communication channel is opened and tested. After that, the firmware version of the drive is checked to prevent any errors. (Once a customer tested and approved the engineering samples received, the firmware in this product will not be changed without permission of the customer. Therefore, every product can have a specific firmware version.)

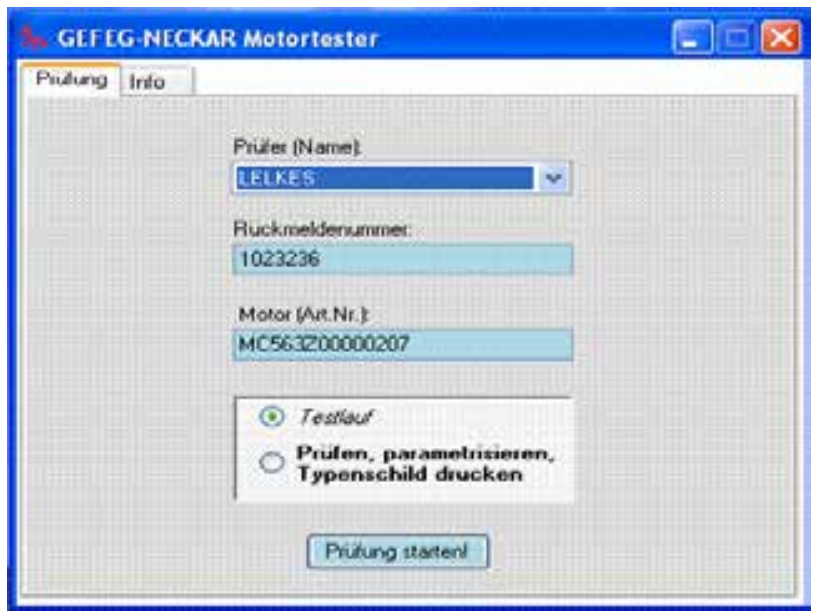

Figure 11. Graphical user interface of the automatic inspection system

In the next step, the information provided by the sensors integrated in the motor is checked. Incorrect Hall-signals, unrealistic temperature values, incorrect voltage values, and dc current values outside the tolerance range can be detected.

If the sensors work properly, the digital inputs and outputs and the analog input processed by the 12 bit analog-digital-converter of the microcontroller are tested individually.

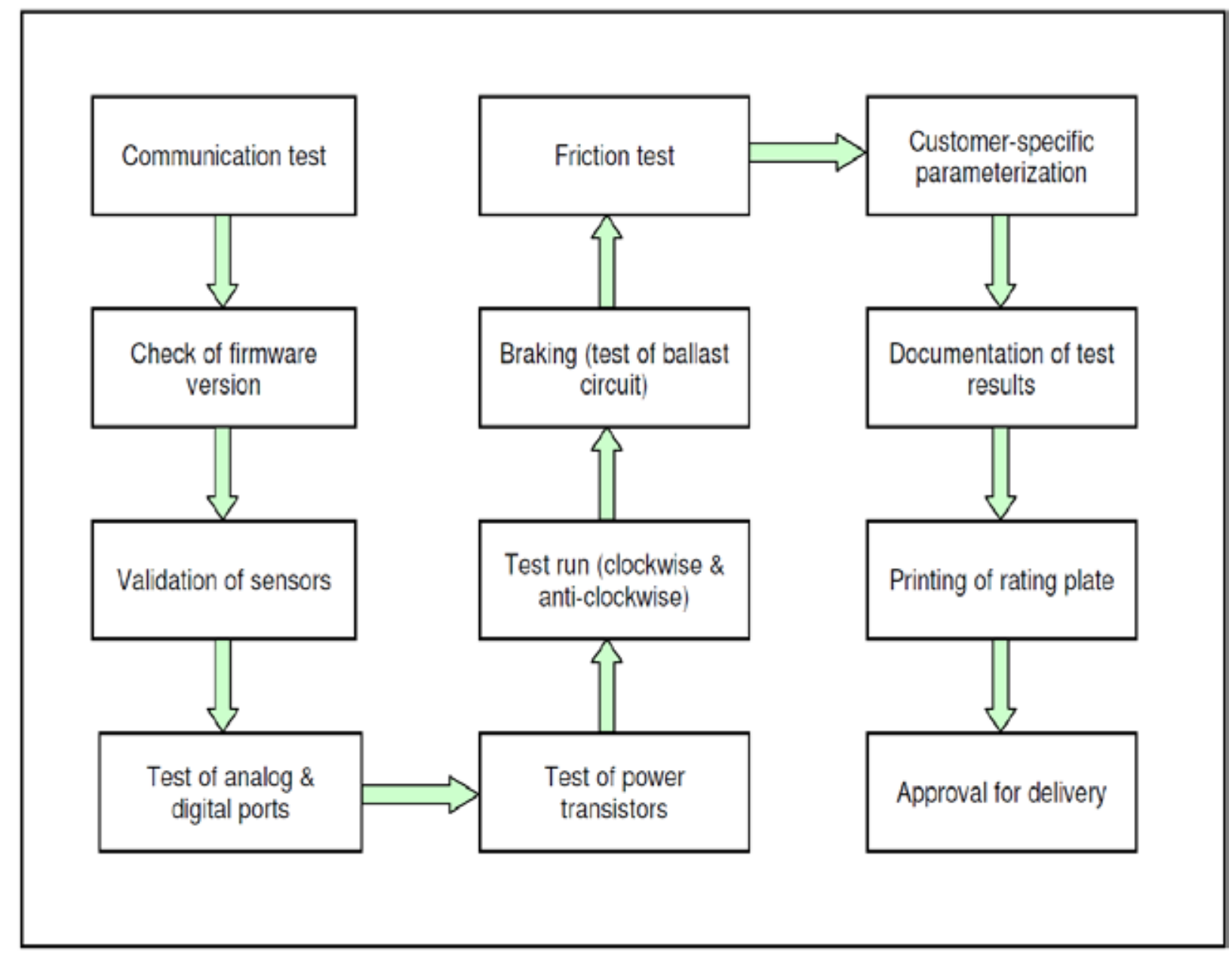

Figure 12. Steps of the test procedure 
Before starting the motor, the power stage of the control unit is tested. In this mode, the control unit works similar to a motor controller for stepper motors. After 5 steps, all the power switches (MOSFETs or IGBTs) will have been tested.

For the test run, the motor is operated in open-loop speed control mode with 100\% PWM factor. Clockwise and anti-clockwise, speed and motor current are monitored and analyzed. From maximum speed, the motor will be slowed down to standstill. In doing so, the ballast circuit can be examined. As a last test step, the motor is accelerated to maximum speed and the power stage is disabled. The motor will coast. Analyzing the deceleration of the rotor, friction in the bearing system and in the gear can be tested.

After a successful test, the measurement data will be archived and the motor will be parameterized according to the agreement with the customer about settings on delivery. The parameter set also contains an individual test number for perfect traceability. Then the rating plate with product name, part number, test number and nominal data is printed as an adhesive label and the motor is ready for delivery.

\section{Conclusions}

An automatic test system for small power electric motors with integrated CANopen ${ }^{\circledR}$ interface was developed. The testing concept utilizes the sensors integrated in the motor and the communication capabilities of the drive. With this inspection system, the produced motors can be tested very fast but thoroughly during manufacturing without the need for cost intensive test stands. An additional advantage of this test method is that it can be easily integrated into the one-piece-flow production system

\section{REFERENCES}

[1] B. Singh, S. Singh, "State of the art on permanent magnet brushless DC motor drives,” Journal of Power Electronics, vol. 9, JPE9-1-1, 2010.
[2] D. Iles, "Small permanent magnet synchronous motor technology - an overview," International Conference for Power Electronics, Intelligent Motion, Power Quality (PCIM), Nuremberg 2008.

[3] A. Binder, "Potentials for energy saving with modern drive technology - a survey," International Symposium on Power Electronics, Electrical Drives, Automation and Motion (SPEEDAM), Ischia 2008.

[4] CiA 402-1/-3, "CANopen Device Profile for Drives and Motion Control," CAN in Automation, Erlangen.

[5] S. Nandi, H.A. Toliyat, L. Xiaodong, "Condition monitoring and fault diagnosis of electrical motors - a review," IEEE Trans. On Energy Conversion, vol. 20, pp. 719-729, 2005.

[6] S. Rajagopalan, T.G. Habetler, R.G. Harley, J.M. Aller, J.A. Respetro, "Diagnosis of rotor faults in brushless DC motors operating under non-stationary conditions using windowed Fourier ridges," Industry Applications Conference, IEEE 2005.

[7] J.-R.R. Ruiz, J.A. Rosero, A.G. Espinosa, L. Romeral, "Detection of demagnetization faults in permanent-magnet synchronous motors under nonstationary conditions," IEEE Trans. on Magnetics, vol. 45, pp 2961-2969.

[8] H. Bae, S. Kim, G. Vachtsevanos, "Fault detection and diagnosis of winding short in BLDC motors based on fuzzy similarity,” International Journal of Fuzzy Logic and Intelligent Systems, vol. 9, pp. 65-162, 2009

[9] R.I. Lörincz, M.E. Basch, I. Bogdanov, V. Tiponut, A. Beschieru, "Hardware implementation of BLDC motor and control system diagnosis,” International Journal of Circuits, Systems and Signal Processing, vol. 5, pp. 660-671, 2011.

[10] B.-G. Park, K.-J. Lee, R.-Y. Kim, D.-S. Hyun, "Low-cost fault diagnosis algorithm for switch open-damage in BLDC motor drives," Journal of Power Electronics, vol. 10, JPE-10-6-17, 2010.

[11] K.-W. Lee, J. Hong, S.B. Lee, S. Lee, “Quality assurance testing for magnetization quality assessment of BLDC motors used in compressors,” IEEE Trans. on Industry Applications, vol. 46, pp. 2452-2458, 2010.

[12] J. Nandan, R. Gobbi, "High reliability for electric machines driving critical loads - a review," Applied Power Electronics Colloquium (IAPEC), IEEE 2011. 